\title{
COMPARISON BETWEEN HAAR WAVELET TRANSFORM, DCT AND A PROPOSED COLUMN- MEAN- METHOD BASED IRIS ENCODERS*
}

\author{
Ibrahim Zedan, Mira M. Sobhi ${ }^{+}$ \\ computers and systems eng. Dept., Faculty of Eng., Zagazig Univ., Zagazig, Egypt.
}

\begin{abstract}
:
Human iris is one of the most reliable biometrics because of its uniqueness, stability and noninvasive nature. Thus it has attracted the attention of biometrics based identification and verification research and development community. Iris recognition has been a fast growing, challenging and interesting area in real-time applications. A large number of iris recognition algorithms have been developed for decades. This paper presents a comparative study of the performance from the iris authentication using Discrete Cosine Transform (DCT), Haar wavelet transform and a proposed Column Mean Method based features. Here iris recognition is done using the image feature set extracted from DCT, Haar Wavelet transform and Column Mean. Analysis was performed with the mentioned methods, consisting of the False Acceptance Rate (FAR) and the Genuine Acceptance Rate (GAR). The proposed technique is tested on an iris image database having 384 images. The iris recognition systems that produce very low error rates were successfully designed. DCT and Column Mean Methods give better performance with the accuracy of $98.44 \%$ and Haar Wavelet Transform gives a performance with the accuracy of $97.66 \%$. The proposed Column Mean Method is superior in having faster recognition rate.
\end{abstract}

KEY WORDS: Iris recognition, biometrics, DCT, Haar Wavelet Transform, Column Mean Method.

\section{COMPARAISON ENTRE LA TRANSFORMEE EN ONDELETTES DE HAAR, DCT ET UNE COLONNE PROPOSE MOYENNES METHODE BASEE IRIS ENCODEURS}

\section{RÉSUMÉ:}

Iris humain est l'un des biométrique la plus fiable en raison de son caractère unique, la stabilité et la nature non invasive . Ainsi, il a attiré l'attention de la biométrie communauté de recherche et développement identification et la vérification sur la base . Reconnaissance de l'iris a été , un domaine difficile et intéressant croissance rapide dans les applications en temps réel . Un grand nombre d' algorithmes de reconnaissance de l'iris ont été développés depuis des décennies. Cet article présente une étude comparative des performances de l'authentification de l'iris à l'aide transformée en cosinus discrète ( DCT ), transformée en ondelettes de Haar et une colonne proposé méthode de la moyenne Les fonctionnalités basées . Voici reconnaissance de l'iris est effectuée en utilisant l'ensemble des fonctionnalités de l'image extraite de DCT , Haar ondelettes transformer et la colonne moyenne. L'analyse a été effectuée avec les méthodes mentionnées , composé du taux des acceptations False ( FAR) et le taux des acceptations véritable ( GAR ). La technique proposée est testée sur une base de données d'image de l'iris ayant 384 images. Les systèmes de reconnaissance de l'iris qui produisent des taux d'erreur très faibles ont été conçus avec succès. DCT et la colonne Méthodes moyennes donnent de meilleures performances avec la précision de 98,44 \% et Haar ondelettes donne une performance avec la précision de 97,66 \% . La colonne proposé Méthode moyen est supérieur en ayant un taux de reconnaissance plus rapide .

MOTS CLÉS: reconnaissance de l'iris , la biométrie , DCT , Haar transformée en ondelettes , Colonne méthode de la moyenne

* Received: 23/12/2012, accepted: 2/4/2014, Ref. No. 132, (Original Paper)

+ Contact author . 


\section{INTRODUCTION}

Identification of humans is a goal as ancient as humanity itself. As technology and services are advanced and developed there is a rapid need for personal identification. Examples include passport control, computer login control, bank automatic teller machines and other transactions authorization, premises access control, and security systems generally. All such identification efforts share the common goals of speed, reliability and automation.

Biometrics as form of unique identification is one of the subjects of researches that are growing more rapidly. There are two types of biometric features: physiological (e.g. iris, face, and fingerprint) and behavioral (e.g. voice and handwriting). The advantages of unique identification using biometrics features are numerous, such as fraud prevention and ease of use. Different biometric feature offer different degrees of reliability and performance.

One of the important techniques used in security systems is iris recognition.

The purpose of 'Iris Recognition', a biometrical based technology for personal identification and verification, is to recognize a person from his/her iris prints. In fact, iris patterns are characterized by high level of stability and distinctiveness. Each individual has a unique iris (see Fig (1)); the difference even exists between identical twins and between the left and right eye of the same person (Ref. (22)).

Iris recognition is the analysis of the colored ring that surrounds the pupil (Ref. (9)). Iris is the annular ring between pupil and sclera of eye. The sclera is the white region of connective tissues surrounding iris. The pupil is the darkest region in eye. The iris is highly protected and non-invasive. The iris has unique structure and these patterns are randomly distributed, which can be used for identification of human being. This makes it comparatively more reliable and stable for identification than the other biometric features such as face and voice (Ref. (21)). With the development of highly sophisticated techniques for iris image acquisition, iris recognition is expected to become an important and widely used form of identification. Its applications range from national identification card, biometric passport and forensics to banking, e-commerce, welfare distribution, etc.

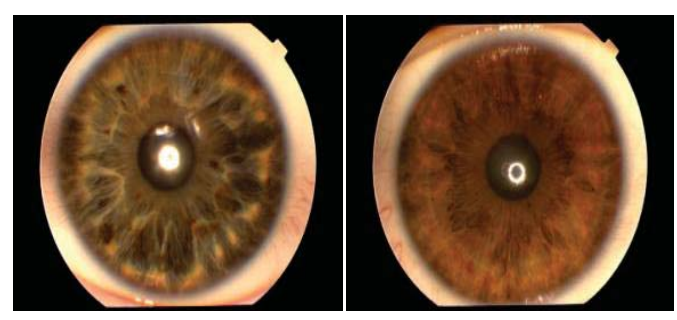

Fig (1): Distinctiveness of human iris

\section{REVIEW OF LITERATURE}

The iris identification using analysis of the iris texture has attracted a lot of attention and researchers have presented a variety of approaches in the literature. Daugman's system is the first known algorithm for iris recognition (Ref. (4)). Daugman has presented most promising 2D Gabor filter based approach for the iris identification system that employed 2048 bit iriscode. It consists of the following major stages:

- Pre-processing stage: To detect the edges of the pupil and iris and to locate the position of the iris within the image with an integro-differential operator.

- Feature extraction stage: To extract iris image pattern using bi-dimensional Wavelets

- Feature classification stage: To perform the classification process with the XOR function applied to the iris code.

Wildes (Ref. (22)) proposed the algorithm which first converts image into a binary edge map and then detects circle using Hough transform. Laplacian filter at multiple scales is used to extract features. Finally, the matching between two iris images is done using normalized correlation. Huang et al. (Ref. (7)) have demonstrated the usage of phase-based local 
correlations for matching iris patterns and ach ieved notable performance over the prior techniques. Proença and Alexandre (Ref. (16)) have suggested region-based feature extraction for the iris images acquired from large distances. S'anchez-Reillo (Ref. (17)) used the left and right portion of the iris in order to avoid the missing data due to eye lashes along with Gabor filters for feature extraction. Liu Yang (Ref. (23)) encrypted the iris code using one way coupled map lattice in order to protect stored template data. Zhonghua Lin and Bibo Lu (Ref. (13)) used the imaginary coefficients of Morlet Wavelet Transform at different scales to generate the binary code of the iris image. Boles (Ref. (2)) has detailed fine-tocoarse approximation at different resolution levels that are based on zero-crossing representation from the wavelet transform decomposition. Jing Huang et al., (Ref. (8)) proposed iris recognition based on non separable wavelet. After decomposing iris image into wavelet sub band coefficients using sixteen non-separable wavelet filters, Generalized Gaussian Density (GGD) modeling of each non separable orthogonal wavelet coefficient was carried for feature extraction. The Kullback Leiblar distance between GGDs was computed for matching. Mohammed Abdullah (Ref. (1)) presented an algorithm using wavelet transform for iris recognition where the feature vector is stored in the form of binary code. Thornton et al. (Ref. (20)) have recently estimated the non-linear deformations from the iris patterns and proposed a Bayesian approach for reliable performance improvement. . Li Ma et al. (Ref. (14)), (Ref. (15)) employed multi-scale bandpass decomposition and evaluated comparative performance from prior approaches.

All above mentioned techniques are computationally intensive and have a large size of the template database formed by the extracted feature vectors.

\section{IRIS PRE-PROCESSING}

The acquired image always contains not only the 'useful' parts (iris) but also some 'irrelevant' parts (e.g. eyelid, pupil etc.). Under some conditions, the brightness is not uniformly distributed. In addition, different eye-to-camera distance may result in different image sizes of the same eye. For the purpose of analysis, the original image needs to be preprocessed. The preprocessing is composed of three steps.

\subsection{Iris Localization}

The segmentation module locates the position of the iris within the image by isolating it from the sclera, pupil, eyelids, and eyelashes.

The task consists of localizing the inner and outer boundaries of the iris. Both are circular, but the problem lies in the fact that they are not cocentric, therefore the two circles must be calculated separately. To do this, a circle detection variant of the normally line detecting Houghtransformation is applied.

\subsection{Iris Normalization}

Two images of the same iris might be very different as a result of: The size of the image, Size of the pupil, Orientation of the iris. To cope with this, the image is normalized by converting from cartesian to doubly dimensionless polar reference form. (Fig (2))

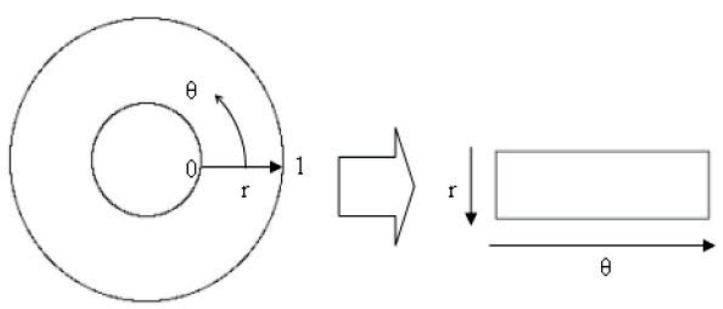

Fig (2) Unwrapping the iris

\subsection{Image Enhancement}

The original iris image has low contrast and may have non-uniform illumination caused by the position of the light source. These may impair the result of the texture analysis. We enhance the iris image and reduce the effect of non-uniform illumination by means of local histogram equalization.

Fig (3) shows the iris preprocessing results. 


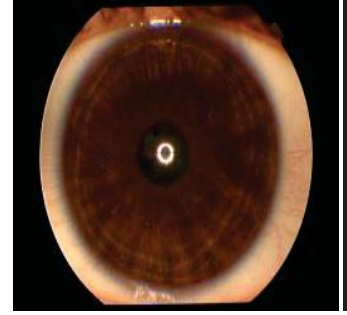

(a)

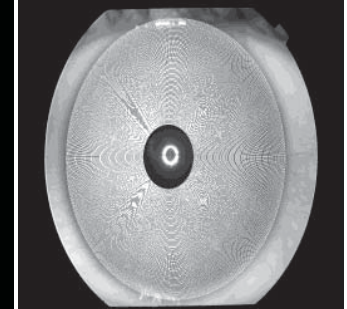

(b)

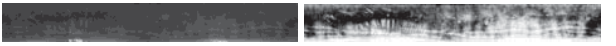

(c)

(d)

Fig (3): Preprocessing (a) Source image. (b) Localized iris and the normalization region used for recognition. (c) Normalized image. (d) Enhanced normalized image.

\section{PROPOSED APPROACH}

\subsection{Haar Wavelet Transform}

This section illustrates the technique of how to get the feature vector (iris code) to be able to compare the similarities of the human eyes and to identify the person.

Wavelet transform is typically used for analyzing the human iris patterns and extracting feature points from them. In this paper, a wavelet transform is used to extract features from iris images. Among the mother wavelets, we use Haar wavelet. The wavelet transform breaks an image down into four sub-sampled, or sub-images. The results consist of one image that has been high passed in the horizontal and vertical directions $(\mathrm{HH})$, one that has been low passed in the vertical and high passed in the horizontal (LH), one that has been high passed in the vertical and low

HH corresponding to filters $g^{t} g, g^{t} h, h^{t} g$, and $h^{t} h$, respectively. The recursive application of this decomposition is used to construct higher level decomposition.

In this approach, the enhanced images are decomposed into five levels by the Haar wavelets. Next the vertical, horizontal and diagonal coefficients of 4th and 5th levels were employed. The coefficients of 1st, 2nd, and 3rd levels were passed in the horizontal (HL) and last that has been low pass filtered in both directions (LL).
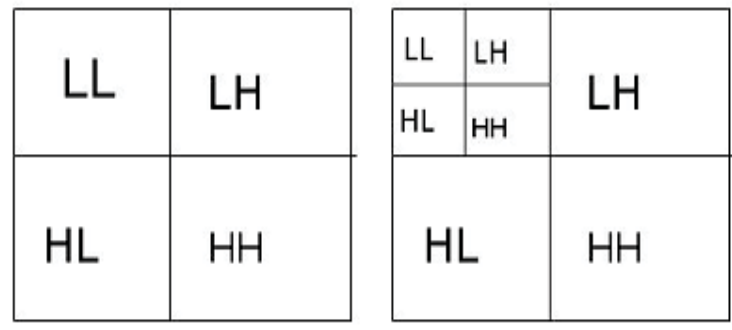

Fig (4): Harr transform

Fig (4) shows the result of Harr transform. Where, $\mathrm{H}$ and $\mathrm{L}$ mean the high pass and low pass filter, respectively. While $\mathrm{HH}$ means that the high pass filter is applied to signals of both directions and represents diagonal features of the image, HL corresponds to horizontal structures, $\mathrm{LH}$ results vertical information and LL corresponds to an approximation image that is further decomposed.

The texture details in the iris region can be analyzed at different resolutions using its multiscale wavelet decomposition. The Haar wavelets can capture sharp discontinuities in the spatial gray-level texture by repeated application of following low-pass (g) and high-pass (h) filters (Ref. (19)):

$$
g=\frac{1}{\sqrt{2}}\left[\begin{array}{ll}
1 & 1
\end{array}\right], h=\frac{1}{\sqrt{2}}\left[\begin{array}{ll}
1 & -1
\end{array}\right]
$$

The above filters are separately applied to the rows and columns of the iris images resulting in four channel filter bank with channels LL, LH, HL, and

almost the same as those of the 4th level and therefore the smallest of them (4th level coefficients) were employed and the rest were ignored. The 5th level decomposition offered the most discriminative information and therefore all the coefficients from this decomposition were employed. The coefficients formed the feature vector. The size of this feature vector was 3 times the size of features of 4 th level $(4 * 32)$ plus 3 times the features of 5 th level $(2 * 16)$, therefore in 
total the size of feature vector was 480 bits. The Euclidean distance was then employed to

ascertain the matching distance between feature vectors.

\subsection{Discrete Cosine Transform (DCT)}

The discrete cosine transform (DCT) (Ref. (12)) represents an image as a sum of sinusoids of varying magnitudes and frequencies. The DCT function for a 2-D image $\left(\mathrm{N}^{*} \mathrm{M}\right)$ is given by Equation (1) and (2)

$$
\begin{aligned}
& B_{M}=a_{p} a_{0} \sum_{m=0}^{N-1} \sum_{m=1}^{1-1} A_{m} \cos \frac{t(2 m+1)}{2 N} \cos \frac{2(2 n+1) \varphi}{2 N} \\
& \alpha_{p}=\frac{1}{\sqrt{\pi}} \text { if } \mathrm{p}=0 \quad \alpha_{\mathrm{q}}=\frac{1}{\sqrt{\pi}} \text { if } \mathrm{q}=0 \\
& =\sqrt{\frac{2}{M}} \text { if } 1 \leq p \leq M-1 \quad=\sqrt{\frac{2}{N}} \text { if } 1 \leq q \leq N-1 \text {. }
\end{aligned}
$$

Where $\mathrm{B}_{\mathrm{pq}}$ are called the DCT coefficients of A which can be an image data $A(m, n)$. The DCT decomposes a signal into its elementary frequency components.

When applied to a $\mathrm{MxN}$ matrix, the 2D-DCT compresses all the energy information of the image and concentrates it in a few coefficients located in the upper-left corner of the resulting $\mathrm{MxN}$ DCT/frequency matrix (Ref. (18)).

Discrete Cosine Transform concentrates the energy coefficient of the image in the upper left corner of the transformed matrix. Hence, we propose a system which uses only these upper-left coefficients and discards the remaining coefficients. These fractional coefficients are further studied to find the optimum fraction that will result in the highest accuracy. It should also be noted that these fractional coefficients may reduce the complexity of the system as the number of coefficients will reduce as the size of the fractions reduce.
For $8 \times 8$ pixel block we obtain 64 DCT coefficients divided into high and low frequencies. Contrary to the high frequency coefficients, the low frequency coefficients have a great effect on image reconstruction. Moreover, after a quantization process, most of the high frequency coefficients are likely to be zero especially for low bit-rate coding. Therefore it becomes interesting to predict these coefficients and to discard them in the DCT calculation and in the quantization process.

The choice of an $8 * 8$ block-size is a trade-off between the need to use a large picture area for the transform, so the energy compaction described above is most efficient, and the fact that the content and movement of the picture varies spatially, which would tend to support a smaller block-size.

In our case for normalized image, horizontal picture dimension is 512 pixels and vertical picture dimension is 64 lines. The number of blocks in one field is $(512: 8) *(64: 8)=64 * 8=512$ macroblocks.

These 512 two-dimensional ( $8 * 8)$ blocks are each input into a DCT that maps the sampled values onto corresponding values in the frequency domain. Each coefficient in the $8 * 8$ DCT domain block indicates the contribution of a different DCT "basis" function to the original image block. The lowest frequency basis function (top-left) is called the DC coefficient and may be thought of as representing the average brightness of the block.

In this approach the DCT transforms a block of 64 intensity values into a block of 6 coefficients ( 3 coefficients vertically and 3 coefficients horizontally) as shown in fig (5). The top left coefficient represents the DC level, or average intensity of the block. 


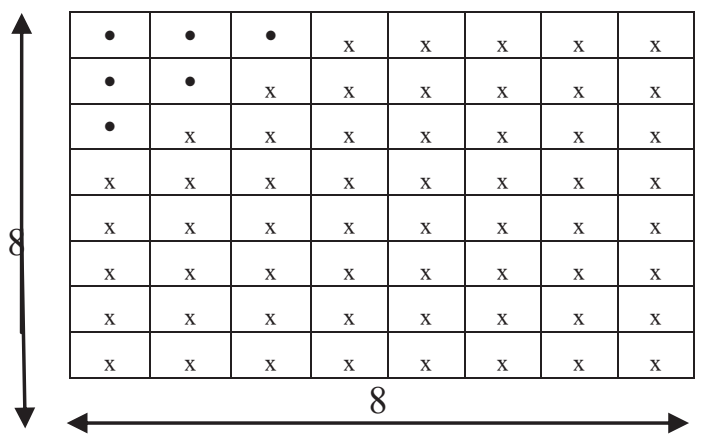

Fig (5): bit reduction in one block

Then the feature vector will be $512 \times 6=3072$ bit.

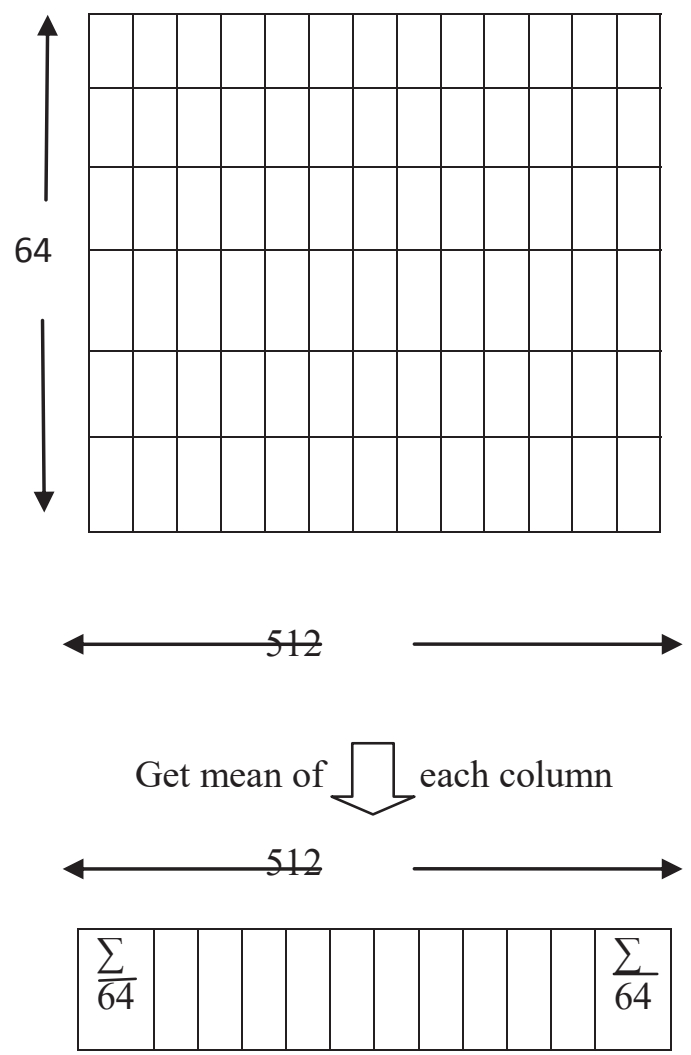

Fig (6): The feature vector of the image

\subsection{The proposed Column Mean Method}

This method is very simple as compared with the other two. It just requires computing the mean of each column in the normalized image. The resulting column means are considered feature vector of iris image. A matching between a query image and stored database images may be performed on the basis of Euclidean Distance between them. Therefore the method is confined to the following two steps: (i) Get the mean of each column in the normalized image to produce a vector of size $(1 \times 512)$ and (ii) store it. This is the $\mathrm{FV}$ of that image as shown in fig (6).

\section{ALGORITHM}

1) Read the database RGB image (Size $=576 \times 768)$.

2) Convert RGB image to gray level image.

3) Detect the edges of the pupil and iris, to locate the position of the iris within the image (localization) then isolate iris region.

4) The isolated area is transformed to a rectangular block of fixed dimension and then normalized (normalization size $=64 \times 512$ )

5) Apply Haar wavelet / dct / mean to each image and store wavelet / dct / mean coefficients in vectors with size of (480/6144/512) (feature vector). This is the FV of that image.

6) Repeat steps 1) through 4) for every database image.

7) Read the query image.

8) Repeat step 2), 3) and 4) for the query image so as to obtain its FV.

9) For every database image ' $i$ ' and a query image ' $q$ ', compute The Euclidean distance (ED) using the following formula:

$$
\mathrm{ED}=\sqrt{(}\left(\sum_{1}^{\mathrm{N}}(\mathrm{FVi}-\mathrm{FVq})^{2}\right)
$$

10) Determine the image with minimum ED and this corresponds to the matching. 


\section{TEMPLATE MATCHING}

The Euclidean distance on the feature set was used as a similarity measure. The direct Euclidian Distance between the database image $\mathrm{P}$ and the test image $\mathrm{Q}$ can be given as equation (3), where $\mathrm{V}_{\mathrm{pi}}$ and $\mathrm{V}_{\mathrm{qi}}$ are the feature vectors of the database image $\mathrm{P}$ and the test image $\mathrm{Q}$ (query) respectively and each is of size " $n "$.

$$
E D=\sqrt{\sum_{i=1}^{n}\left(V_{p i}-V_{q i}\right)^{(63)}}
$$

\section{IMPLEMENTATION}

\subsection{Platform}

The experiments were performed with a Matlab R2010b, on an Intel Core 2 Duo T5870 (2 Ghz) computer.

\subsection{Database}

The Phoenix database (Ref. (3)) consists of 6 images (3-right eye and 3-left eye) for each of 64 individuals (total of 384 images). Samples of six images taken per person for three people are shown in Fig (7).

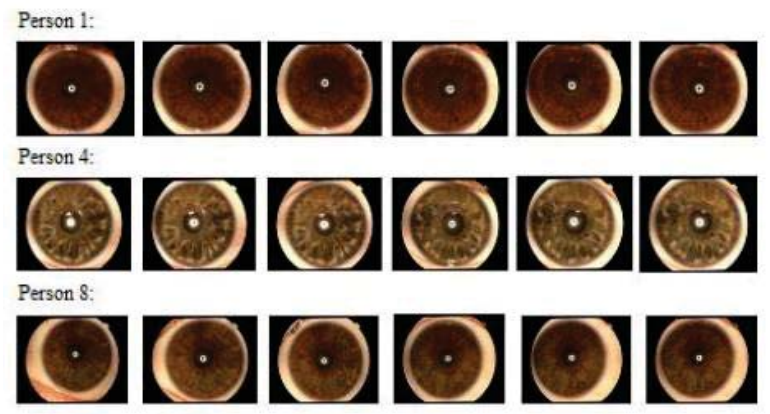

Fig (7) Sample Images from Iris Database

Out of these 6 images of each individual, 4 images i.e. 2 of the left eye and 2 of the right eye were used as training set and the remaining images i.e. 1 of the left eye and 1 of the right eye were used for testing. Thus, the training set constituted 256 images and the test data constitute of 128 images (ratio 2:1).

\section{RESULTS \& DISCUSSIONS}

The false acceptance rate (FAR) (Ref. (6)) is a measure of the likelihood that the biometric

security system will incorrectly accept an access attempt by an unauthorized user. A system's FAR typically is stated as the ratio of the number of false acceptances divided by the number of identification attempts.

The genuine acceptance rate (GAR) (Ref. (5)) is a measure of the likelihood that the biometric security system will correctly accept an access attempt by an authorized user. A system's GAR typically is stated as the ratio of the number of correct acceptance divided by the number of identification attempts.

The FAR and GAR values were calculated for the comparison of all the test images with all images in the database.

A Threshold must be determined to determine best accuracy and smallest FAR. If the Euclidean distance between two templates is less than the threshold, the templates were generated from the same iris and a match is found. Otherwise if the Euclidean distance is greater than the threshold, the two templates are considered to have been generated from different irises.

Fig (8), (9), (10) show the identification accuracy obtained for Haar wavelet, Column Mean Method and DCT.

The highest accuracy obtained for Haar wavelet as shown in Fig (8) occurs at threshold equal 4.9. At this threshold GAR equal 97.66 and FAR equal 2.34 .

The highest accuracy obtained for Column Mean Method as shown in Fig (9) occurs at threshold equal 4.7. At this threshold GAR equal 98.44 and FAR equal 1.56.

The highest accuracy obtained for DCT as shown in Fig (10) occurs at threshold equal 4.4. At this threshold GAR equal 98.44 and FAR equal 1.56. 


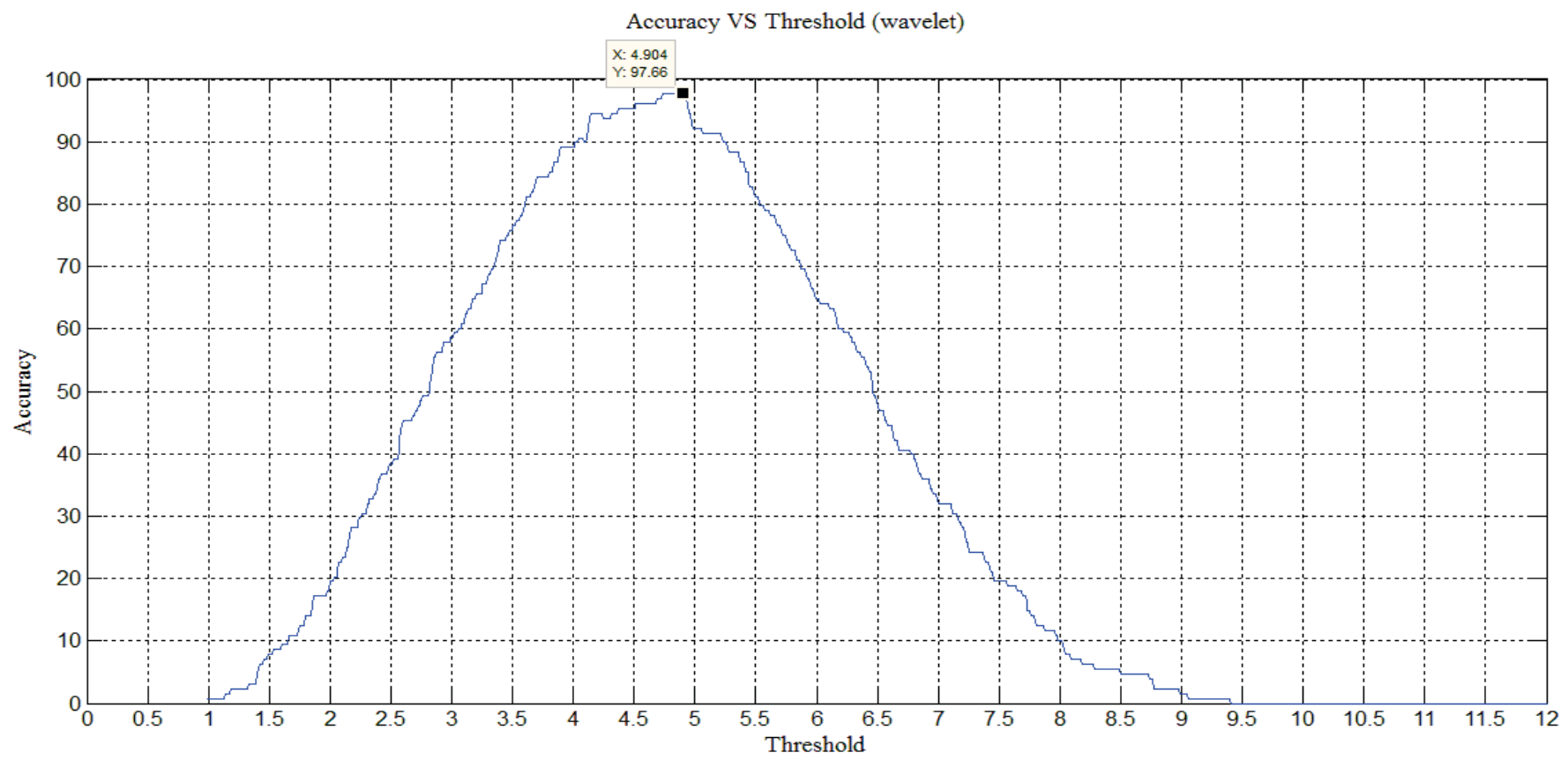

Fig (8) Identification accuracy obtained for Haar wavelet

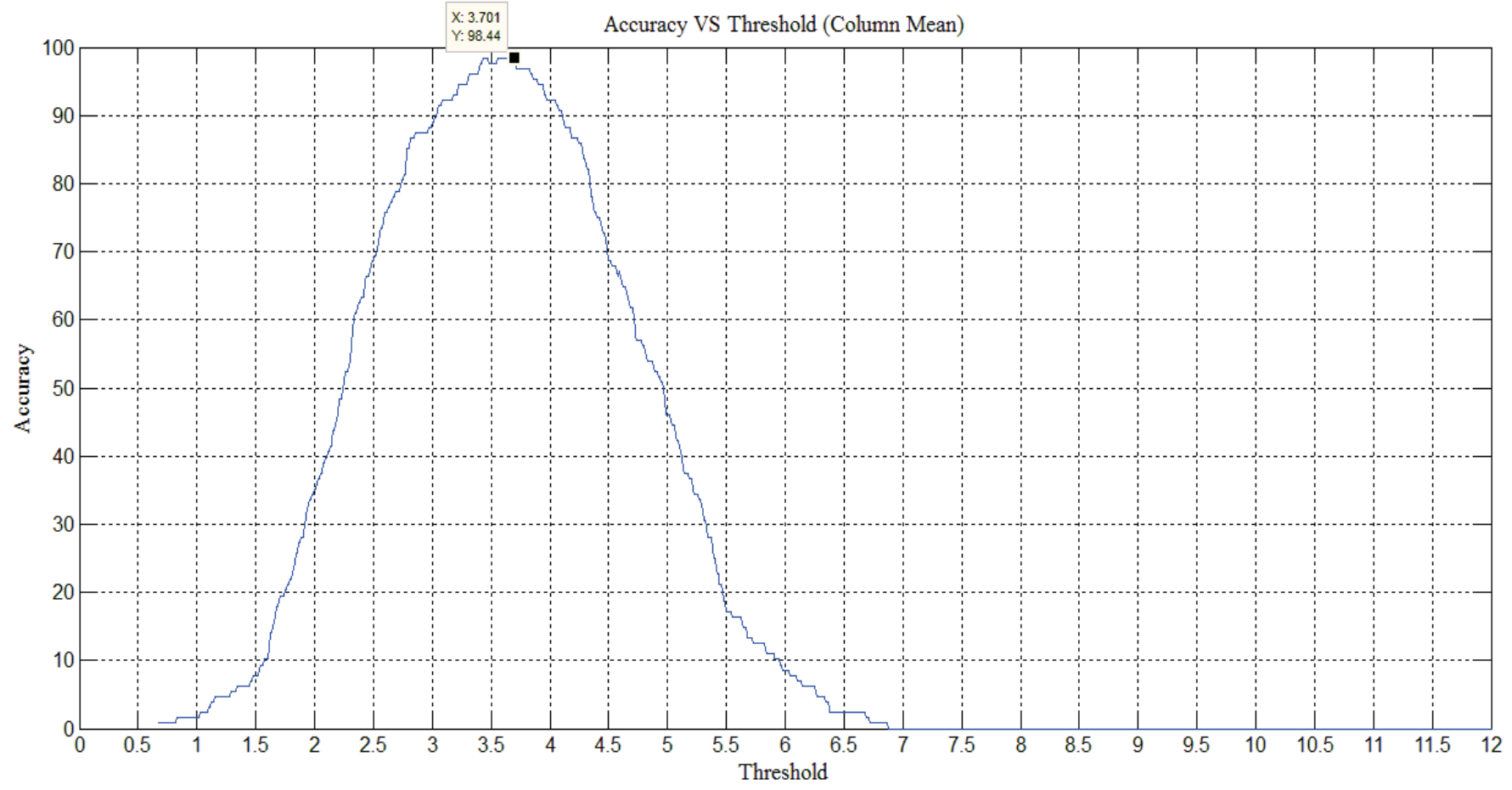

Fig (9) Identification accuracy obtained for Column Mean Metho 


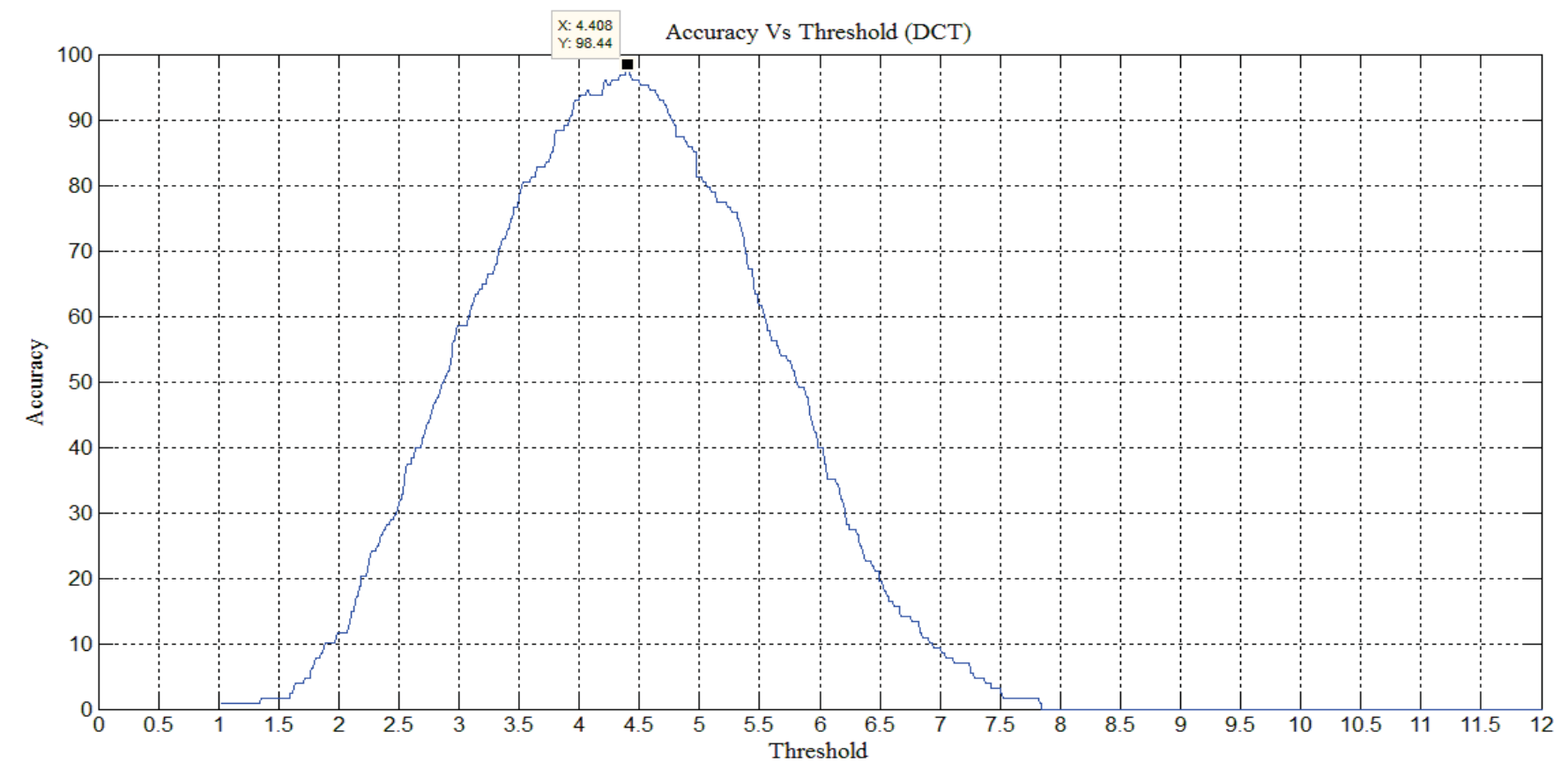

Fig (10) Identification accuracy obtained for DCT

\section{CONCLUSIONS}

This paper has investigated a comparative performance between three different approaches for the iris identification: DCT, Haar wavelet transform and Column mean Method. Experimental results show that the performance of the DCT and Column Mean Method are the same and better than the Haar wavelet.

An accuracy of $98.44 \%$ for DCT, $97.66 \%$ for Haar wavelet and $98.44 \%$ for Column Mean Method. We obtained Column Mean Method and DCT have the same accuracy, but the Column Mean Method is easy to compute and fast compared to the other two methods on texture analysis. Also the Feature Vector obtained from Column Mean Method (size 512) is smaller than that of DCT (size 3072), The reduced feature vector size provides faster recognition rate.

Kekre H. et al. (Ref. (11)) proposed an approach for the iris identification using the five level Haar wavelet transform on the same database, but the accuracy rate was very poor( $64.5833 \%)$.
Also the approach proposed by Kekre H. et al. (Ref. (10)) showed an iris recognition using DCT with accuracy $79.6875 \%$ and Haar wavelet with accuracy $80.4688 \%$. No pre-processing of the images was done to separate the iris in those papers; this may be the reason for poor accuracy.

Finally, Column Mean Method is giving better performance than other methods (Dct and wavelet).

\section{REFERENCES}

[1] Abdullah, M. A., Al-Dulaimi, F. H. , Al-Nuaimy, W. and Al-Ataby, A., (2011) "Smart Card with Iris Recognition for High Security Access Environment, " IEEE International Conference on Biomedical Engineering, pp. 382-385,

[2] Boles, W. W., (1997) "A security system based on human iris identification using wavelet transform", Proc. Intl. Conf. Knowledge-Based Intelligent Electronic Systems, pp. 533-541.

[3] Database:Iris database is available on http://phoenix.inf.upol.cz/iris/download/ 
[4] Daugman, J.,( 1993 ) "High confidence visual recognition of persons by a test of statistical independence ", IEEE Transactions on Pattern Analysis and Machine Intelligence, vol. 15, no. 11, pp. 1148-1161,.

[5]http://www.webopedia.com/TERM/F/false_accep tance.html

[6]http://www.webopedia.com/TERM/F/genuine_ac ceptance.html

[7] Huang, J., Tan, T., Ma L., Wang Y.,(2005) "Phase correlation based iris image registration model, " Computer Science and Technology, vol. 20, no. 3, pp. 419-425.

[8] Huang, J., You, X., Tang, Y. Y., (2008) "Iris Recognition Based on Non Separable Wavelet", IEEE International Conference on Systems, Man and Cybernetics, pp. 1552-1557,

[9] Jain, A. K., Flynn, P. and Ross, A., (2008) "Handbook of Biometrics ", New York: Springer.

[10] Kekre H. B., Sarode T. K., Bhatia p., et al.,(2011) " Iris recognition using Partial Coefficients by applying Discrete Cosine Transform, Haar Wavelet and DCT Wavelet Transform " International Journal of Computer Applications (0975 - 8887) Volume 32- No.6,

[11] Kekre H.B., Thepade S.D., Jain j., et al., (2010) "IRIS Recognition using Texture Features Extracted from Haarlet Pyramid" International Journal of Computer Applications (0975 - 8887) Volume 11No.12.

[12] Kekre, H. B., Sarode, T. K., Natu S, an Natu, P., "Performance Comparison Of 2-D DCT On Full/Block Spectrogram And 1-D DCT On Row Mean Of Spectrogram For Speaker identification", International Journal of Biometrics and Bioinformatics, Vol. 4, Issue 3, pp. 100-112.

[13] Lin, Z. and Lu, B. ,(2010) "Iris Recognition Method Based on the Imaginary Coefficients of Morlet wavelet Transform ", Seventh IEEE international Conference on Fuzzy Systems and Knowledge Discovery, pp. 573-577.
[14] Ma, L., Tan, T., Wang, Y. and Zhang, D., (2004) "Efficient Iris Recognition by characterizing Key Local Variations ", IEEE Trans. Image Process., vol. 13, pp. 739-750.

[15] Ma, L., Tan,T., Wang, Y., and Zhang ,D.,(2003) "Personal identification based on iris texture analysis", IEEE Trans. Patt. Anal. Machine Intell., vol. 25, pp. 1519-1533,.

[16] Proença, H. and Alexandre, L. A., (2007) "Towards noncooperative iris recognition: A classification approach using multiple signatures ", IEEE Trans. Patt. Anal. Machine Intell., vol. 29, pp. 607-612.

[17] Sanchez-Reillo, R. and Sanchez-Avila ,C.,( 2001) "Iris recognition with low template size", in AVBPA, ser. Lecture Notes in Computer Science, J. Big"un and F. Smeraldi, Eds., vol. 2091. Springer, pp. 324-329.

[18] Sarhan, A. M., (2009) "Iris Recognition Using Discrete Cosine Transform and Artificial Neural Networks", Journal of Computer Science Vol. 5, No.5, pp.: 369-373.

[19]Stollnitz, E., DeRose, T., Salesin, D., (1996) "Wavelet for Computer Graphics: Theory andApplications ", Morgan Kaufmann, LosAltos, CA.

[20] Thornton, J., Savvides, M., and Vijay Kumar, B. V. K., (2007) "A Bayesian approach to deformed pattern matching of iris images", IEEE Trans. Patt. Anal. Machine Intell., vol. 29, pp. 596-606.

[21] Tisse, C. , Martin, L. , Torres, L. and Robert, M. ,( 2002) "Person Identification Technique using Human Iris Recognition ", Proceedings of Vision Interface, pp. 294-299.

[22]Wildes, R.P, (1997) "Iris Recogntion: An Emerging Biometric Technology", Proceedings of the IEEE, VOL. 85, NO. 9, pp. 1348-1363.

[23] Yang ,L. , Dong, Y. X., Fei, L. Y., and Yan ,H., (2010) "Iris Recognition System Based on Choas Encryption", IEEE International Conference on Computer Design and Applications, vol 1, pp. 537539. 